\title{
Competency level assessment of healthcare practitioners in managing diabetes and diabetic eye disease in the district health system of Limpopo province, South Africa
}

\begin{tabular}{|c|c|}
\hline \multicolumn{2}{|c|}{$\begin{array}{l}\text { Authors: } \\
\text { Zaheera } \text { Abdool }^{1} \text { @ } \\
\text { Kovin Naidoo } \\
\text { Linda Visser }\end{array}$} \\
\hline \multicolumn{2}{|c|}{$\begin{array}{l}\text { Affiliations: } \\
{ }^{1} \text { Department of Optom } \\
\text { Allied Health Support } \\
\text { Services, Voortrekker } \\
\text { Hospital, Mokopane, } \\
\text { South Africa }\end{array}$} \\
\hline \multicolumn{2}{|c|}{$\begin{array}{l}{ }^{2} \text { Department of Optometry, } \\
\text { Faculty of Health Sciences, } \\
\text { University of KwaZulu-Natal, } \\
\text { Durban, South Africa }\end{array}$} \\
\hline \multicolumn{2}{|c|}{$\begin{array}{l}{ }^{3} \text { Department of } \\
\text { Ophthalmology, School of } \\
\text { Clinical Medicine, Nelson R } \\
\text { Mandela School of Medicine, } \\
\text { Durban, South Africa }\end{array}$} \\
\hline \multicolumn{2}{|c|}{$\begin{array}{l}\text { Corresponding author: } \\
\text { Zaheera Abdool, } \\
\text { zaheera.abdool1@gmail.com }\end{array}$} \\
\hline $\begin{array}{l}\text { Dates: } \\
\text { Received: } 30 \\
\text { Accepted: } 19 \\
\text { Published: } 14\end{array}$ & $\begin{array}{l}\text { Aar. } 2020 \\
\text { Aug. } 2020 \\
\text { Dec. } 2020\end{array}$ \\
\hline \multicolumn{2}{|c|}{$\begin{array}{l}\text { How to cite this article: } \\
\text { Abdool Z, Naidoo K, Visser L. } \\
\text { Competency level } \\
\text { assessment of healthcare } \\
\text { practitioners in managing } \\
\text { diabetes and diabetic eye } \\
\text { disease in the district health } \\
\text { system of Limpopo province, } \\
\text { South Africa. Afr Vision Eye } \\
\text { Health. 2020;79(1), a569. } \\
\text { https://doi.org/10.4102/ } \\
\text { aveh.v79i1.569 }\end{array}$} \\
\hline \multicolumn{2}{|c|}{$\begin{array}{l}\text { Copyright: } \\
\text { (c) 2020. The Author(s). } \\
\text { Licensee: AOSIS. This work } \\
\text { is licensed under the } \\
\text { Creative Commons } \\
\text { Attribution License. }\end{array}$} \\
\hline \multicolumn{2}{|l|}{ Read onli } \\
\hline 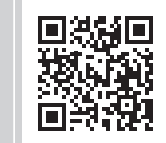 & $\begin{array}{l}\text { Scan this QR } \\
\text { code with your } \\
\text { smart phone or } \\
\text { mobile device } \\
\text { to read online. }\end{array}$ \\
\hline
\end{tabular}

Authors:

Affiliations:

${ }^{1}$ Department of Optometry,

Allied Health Support

Hospital, Mokopane,

${ }^{2}$ Department of Optometry, Faculty of Health Sciences, University of KwaZulu-Natal,

${ }^{3}$ Department of

Durban, South Africa

Corresponding author:

Zaheera Abdool,

Dates:

Received: 30 Mar. 2020

Accepted: 19 Aug. 2020

How to cite this article:

Abdool Z, Naidoo K, Visser L. Competency level practitioners in managing diabetes and diabetic eye disease in the district health system of Limpopo province, https://doi.org/10.4102/ v79i1.569
Background: There are many gaps in the management of diabetes mellitus (DM) and diabetic eye disease in the district health system (DHS) of South Africa (SA). National guidelines recommend annual eye examinations for patients with DM.

Aim: The purpose of this study was to describe the self-reported skill levels of healthcare practitioners (HCPs) to conduct eye examination procedures required for a proposed diabetic retinopathy (DR) screening model.

Setting: The study was conducted in public health institutions of Waterberg district and Mankweng Hospital complex (Capricorn district) in Limpopo province, SA.

Methods: A cross-sectional design using purposive sampling was conducted, and questionnaires were distributed to a total of 74 HCPs. The questionnaires distributed included questions relating to the competency levels of primary healthcare nurses (PHC nurses), optometrists, ophthalmic nurses and medical officers (MOs) regarding examination procedures in the management of patients with DM and whether they agreed with the developed DR screening model.

Results: All the PHC nurses had knowledge about all the examination procedures required in the proposed DR screening model, whilst $94.1 \%$ of MOs exhibited knowledge regarding the procedures required from them. Optometrists lacked knowledge of grading DR, and ophthalmic nurses were least knowledgeable about conducting internal and external eye examinations and in detecting and grading DR.

Conclusion: The proposed DR screening model did not need modification. The involvement of dieticians and more ophthalmic nurses could be beneficial to the DR screening model.

Keywords: healthcare practitioners; diabetes mellitus; diabetic eye disease; diabetic retinopathy; district health system.

\section{Introduction}

Diabetic retinopathy (DR) is a common ocular manifestation of diabetes mellitus (DM), which is potentially sight threatening. ${ }^{1,2,3}$ In its initial stages, DR may be asymptomatic but can progressively cause gradual loss of vision or lead to sudden, irreversible blindness if left undetected. ${ }^{4}$ Surgical treatments are available to treat DR; however, these procedures could further complicate or reduce vision if instituted at an advanced stage in the disease's progression. ${ }^{5}$

Currently, in the district health system (DHS), patients with DM are not referred to optometrists unless they complain of vision problems. The first port of call for patients is at the primary healthcare (PHC) clinics, managed by PHC nurses who are responsible for taking vital signs and a brief case history. Patients with high blood glucose levels (hyperglycaemia) are referred to general practitioners stationed at the hospitals, known as medical officers (MOs), for DM diagnosis and treatment. Referral to other healthcare practitioners (HCPs) is based on patients' chief complaint, which means that patients may not be referred to other HCPs (optometrists, dieticians, physiotherapists, psychologists, dentists, etc.) in the absence of complaining about other symptoms or problems, and thus not all patients with DM are screened for diabetic eye complications. The PHC nurses often educate patients with DM on weight control and foot care 
as part of their routine screening but not on the importance of hyperglycaemic control to prevent vision problems. Also, because of time constraints not all MOs emphasise the importance of glycaemic control to prevent vision problems and the assumption is that the PHC nurses are to provide patient education at the clinics.

With regard to screening for DM and DR, limited resources such as the lack of equipment in clinics and district hospitals and the lack of optometric skills in conducting certain eye examination procedures (e.g. ocular pupil dilations) prevent MOs and optometrists from appropriately referring patients to ophthalmologists. Inappropriate referrals lead to an increase in ophthalmologists' workload and create unnecessary logistical costs and inconvenience for both the patients and the healthcare system. Screening for DR by primary HCPs in Africa has proven to be financially advantageous for both the patient and the healthcare system ${ }^{6}$ but not cost-effective for ophthalmologists to screen and treat DR at the same time.

Clinical practice guidelines for DR diagnosis have been adopted in various countries, but with variations in methods of examinations and also in HCPs involved in screening and diagnosing DR. ${ }^{7}$ Despite the existing guidelines for frequency of referral and grading criteria for DR in South Africa (SA), constraints in the DHS have contributed to a lack of or poor implementation of DR screening programmes., ${ }^{8,9}$ As the progression of DR depends on the duration of $\mathrm{DM},{ }^{10}$ glycaemic control $^{11}$ and the presence of systemic complications, ${ }^{10} \mathrm{DM}$ management is key to reduce the DR progression. This situation, therefore, demands a holistic, multidisciplinary team approach of HCPs to reduce blindness and other associated complications of DM, which would increase DR progression. Each HCP has a specific role to play in the management of DM and DR.

The purpose of this study was to describe the self-reported competency levels of participants involved in DR screening using a DR screening model, which was developed for a DHS (Figure 1).

\section{Methodology}

A purposive sampling strategy was employed whereby all MOs working in Voortrekker district hospital and all PHC nurses from seven out of nine clinics, which are the catchment areas for Voortrekker hospital patients, were selected for participation. All ophthalmic nurses and optometrists in Waterberg district and Mankweng provincial hospital complex were recruited as both hospitals were referral institutions for Voortrekker hospital patients. The total number of participants was 64 (see Table 1).

The inclusion criteria for participants were that HCPs must have been involved in the clinical management of $\mathrm{DM}$ and diabetic eye complications. The exclusion criteria were HCPs who were not qualified to manage diabetic

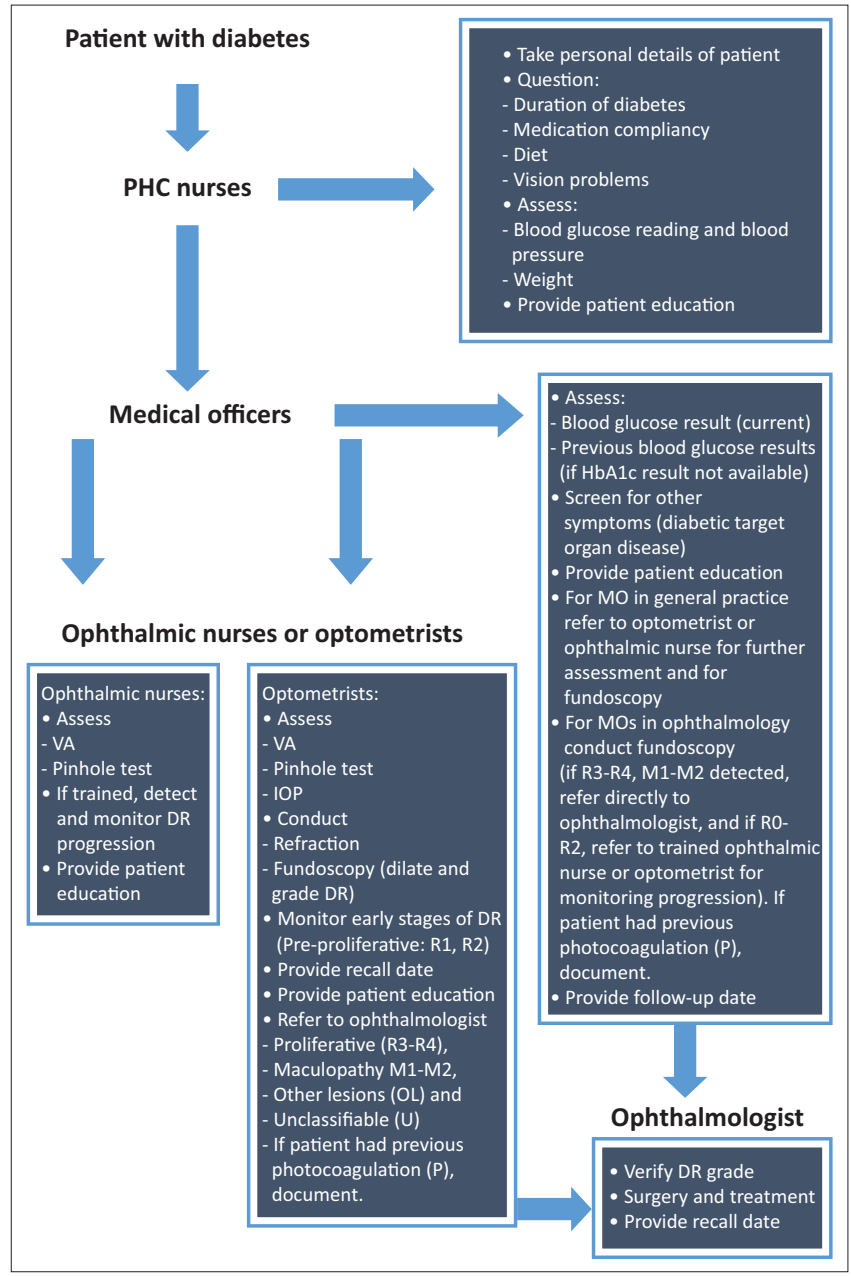

MO, medical officer; DR, diabetic retinopathy; PHC, primary healthcare; IOP, intraocular pressure; VA, visual acuity.

FIGURE 1: Diabetic patient flow diagram.

TABLE 1: Categories and number of participants from clinics and hospitals.

\begin{tabular}{lccc}
\hline Categories & $\begin{array}{c}\text { Total number } \\
\text { of participants }\end{array}$ & $\begin{array}{c}\text { Total number of } \\
\text { recruited participants }\end{array}$ & $\begin{array}{c}\text { Percentage response } \\
\text { rate of participants }\end{array}$ \\
\hline PHC nurses & 14 & 17 & 82.3 \\
MOs & 17 & 17 & 100.0 \\
Ophthalmic nurses & 10 & 15 & 66.7 \\
Optometrists & 23 & 25 & 92.0 \\
\hline Total & $\mathbf{6 4}$ & $\mathbf{7 4}$ & $\mathbf{8 6 . 5}$ \\
\hline
\end{tabular}

PHC, primary healthcare; MO, medical officers.

eye complications. Permission to conduct the study at Mankweng provincial hospital and FH Odendaal was obtained from the Department of Health, Limpopo province and the respective heads of institutions. Information relating to the study was provided and informed consent was obtained from all participants. Questionnaires to PHC nurses, MOs, ophthalmic nurses and optometrists were hand-delivered by the principal investigator. Responses from the participants were analysed using standard frequencies and proportions.

\section{Ethical consideration}

The protocol was submitted to the University of KwaZuluNatal's School of Health Sciences Faculty Review 
Committee and then to the Biomedical Research and Ethics Committee (BREC). Ethical clearance was obtained before commencement of the study (BREC Ref no: 272/18, 29 May 2018).

\section{Results and discussion Primary healthcare nurses}

PHC nurses play an important role in building a network for referral and screening at community level. They bridge the gap between the community and other HCPs as they refer patients with DM to MOs in general practice and those complaining of ocular complications to ophthalmic nurses and optometrists. They are mainly responsible for taking case history, screening vital signs and providing patient education on the management of systemic complications of DM. The role of PHC nurses varies by province as it was established that PHC nurses in KwaZulu-Natal also conducted visual acuity (76.2\%), pinhole testing (57.1\%) and external eye examination $(85.7 \%) .{ }^{1}$ The role of PHC nurses in patient education is invaluable as they can re-emphasise this on every patient visit. In this study, more than $80 \%$ of $\mathrm{PHC}$ nurses educated patients with DM on foot care, diet and glycaemic control (Figure 2). The DR screening model did not require PHC nurses to conduct visual acuity (VA) or other ocular screening tests. The model is therefore conducive to their required role in the management of patients with DM. Only one PHC nurse $(7.1 \%)$ indicated that the DR screening model needed modification but did not indicate reasons for this.

\section{Medical officers}

In a district health setting, MOs in general practice are stationed at hospitals in different sections, namely, outpatients department, casualty, wards and theatre, whilst a

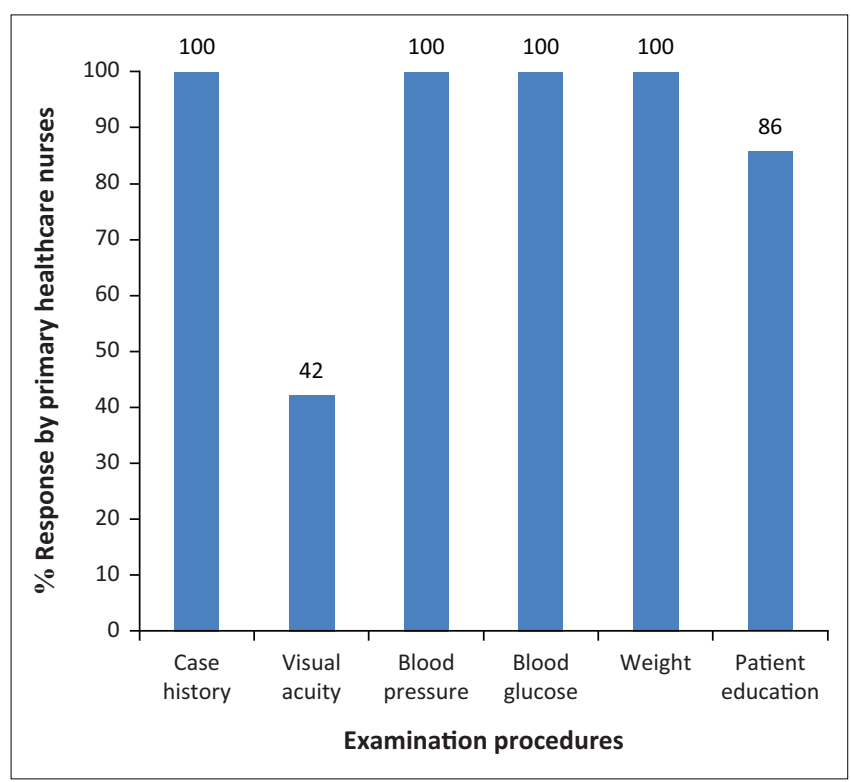

FIGURE 2: Percentage of self-reported competency level of primary healthcare nurses in conducting examination procedures. minority are appointed to conduct outreach services at clinics. Medical officers in ophthalmology are stationed in the ophthalmology unit and work under the supervision of an ophthalmologist, conducting ocular surgical procedures. Medical officers play a key role in detecting and managing the systemic complication of DM, termed diabetic target organ disease, which includes the assessment of the brain, heart, kidneys, arteries and eyes. In the DHS, standard treatment guidelines ${ }^{12,13}$ are available for managing patients with DM. These include diagnosing, monitoring and treating $\mathrm{DM}$ and associated systemic risk factors to prevent microand macro-vascular complications.

As per DR flow diagram (Figure 1), vital signs were recommended to be assessed by nursing staff, and MOs in conjunction with nurses were required to monitor blood glucose levels, weight and blood pressure of patients with DM. Glycaemia control is established by conducting an haemoglobin A1c test, which is ordered on a 3- or 6-month basis as per MO's request. According to the National Department of Health Guidelines, ${ }^{12,13}$ patients with DM are recommended to have a retinal examination on an annual basis, and ophthalmic conditions, which cannot be managed on site such as proliferative DR (PDR) and cataracts, should be referred to ophthalmologists. The guideline, however, does not stipulate who should be the responsible HCPs for conducting the eye screening. At present patients with DM are referred by the PHC nurses from the clinics after assessing vital signs, and depending on the complexity of the complaint, patients are referred to the MOs in various departments and only those complaining of visual complications are referred to optometrists.

Owing to the lack of ophthalmologists in public hospitals, patients requiring referral should be screened for DR and monitored before referral. This helps to reduce the patient backlog, as ophthalmologists are also involved in training MOs in ophthalmology and cannot assess every patient with ocular pathology referred and therefore require optometrists and ophthalmic nurses to monitor DR progression. Medical officers in ophthalmology are usually stationed in regional and provincial hospitals and work directly under the supervision of ophthalmologists. There are no ophthalmic nurses at clinics in Limpopo province to manage anterior segment ocular complications. Optometrists usually screen patients at clinics for ocular complications using an ophthalmoscope on a monthly basis as opposed to MOs in general practice who are allocated to specific clinics on a daily basis. Medical officers generally have limited experience in the use of an ophthalmoscope and in this study only $41.2 \%$ were familiar with conducting fundoscopy (Figure 3). There is therefore a need for protocols to be developed for MOs working at clinics to screen for anterior segment disease to assist with diagnosis of serious ocular pathology, such as iris neovascularisation, which requires urgent referral to an ophthalmologist. 


\section{Ophthalmic nurses}

All ophthalmic nurses are based at district and regional hospitals in Limpopo province. They are an important bridge between the PHC nurses, MOs, optometrists and ophthalmologists. Ophthalmic nurses in district and regional hospitals in Limpopo province have specific roles to play in theatre, wards and clinics assisting ophthalmologists with pre- and post-operative procedures. The roles of ophthalmic nurses generally vary according to the availability of resources (staff, equipment and training). In Ghana, the role of the three ophthalmic nurses working at Bawku Hospital included carrying out pre- and post-management of eye injuries, counselling patients who were irreversibly blind for rehabilitation, educating the public and other HCPs on primary eye care, organising and conducting outreach programmes and managing ocular emergencies. Furthermore, patients with DM were screened for DR as the ophthalmologist only visited the hospital on a monthly basis. ${ }^{14}$

In this study, all the ophthalmic nurses qualified more than 15 years back but only $60 \%$ were practising for the 15 years (Table 2). Knowledge of external and internal eye examination was poor as only one ophthalmic nurse knew how to conduct fundoscopy, but two were trained on how to detect DR and only three had knowledge about detecting iris neovascularisation (Figure 5). It is difficult to encourage and motivate the expansion of ophthalmic nursing roles in Limpopo province because of their scarcity. As such patients requiring visual assessment are referred by the PHC nurses from the clinics to optometrists at the hospitals. All the ophthalmic nurses understood the referral process of the DR screening model and one ophthalmic nurse indicated modification of the model, suggesting that dieticians and more ophthalmic nurses should be employed and dedicated to DM and DR management.

TABLE 2: Age, year of qualification and duration of practice of optometrists and ophthalmic nurses.

\begin{tabular}{|c|c|c|c|c|}
\hline \multirow[t]{2}{*}{ Participant profile } & \multicolumn{2}{|c|}{ Optometrists } & \multicolumn{2}{|c|}{ Ophthalmic nurses } \\
\hline & $n$ & $\%$ & $n$ & $\%$ \\
\hline \multicolumn{5}{|l|}{ Age (years) } \\
\hline $25-30$ & 8 & 34.8 & 0 & - \\
\hline $31-35$ & 9 & 39.1 & 0 & - \\
\hline $36-40$ & 2 & 8.7 & 1 & 10.0 \\
\hline $41-45$ & 4 & 17.4 & 1 & 10.0 \\
\hline$>45$ & - & - & 8 & 80.0 \\
\hline \multicolumn{5}{|l|}{ Year of qualification } \\
\hline 1970-2000 & 3 & 13.0 & 9 & 90.0 \\
\hline 2001-2005 & 2 & 8.7 & 1 & 10.0 \\
\hline 2006-2010 & 6 & 26.1 & 0 & - \\
\hline 2011-2015 & 11 & 47.8 & 0 & - \\
\hline 2016-2020 & 1 & 4.3 & 0 & - \\
\hline \multicolumn{5}{|l|}{ Duration of practice } \\
\hline$<5$ years & 3 & 13.0 & 1 & 10.0 \\
\hline $5-10$ years & 14 & 60.9 & 2 & 20.0 \\
\hline $11-15$ years & 2 & 8.7 & 1 & 10.0 \\
\hline $16-20$ years & 3 & 13.0 & 4 & 40.0 \\
\hline $21-25$ & 1 & 4.3 & 1 & 10.0 \\
\hline$>25$ & 0 & - & 1 & 10.0 \\
\hline
\end{tabular}

\section{Optometrists}

In this study, from all the participants, only one optometrist $(4.3 \%)$ did not understand the proposed protocol and also did not indicate the area of concern (Figure 4). In the management of patients with DM, the role of optometrists in general includes conducting refraction and both external and internal eye examination. However, having insufficient training to detect and classify DR prevents optometrists from appropriately screening, grading, referring and recalling patients who need to be monitored. ${ }^{1}$ Though only $52.2 \%$ had knowledge on DR grading, more than 90\% (91.3\%) knew how to detect DR (Figure 6). Of the 11 optometrists who qualified after 2011, 5 (41.7\%) knew how to grade DR. There was, however, no association found between years of qualification and DR grading ( $p=0.414)$ (Table 3). By having skills to detect and grade DR will reduce the burden on ophthalmologists as optometrists can provide support for their pre- and post-laser treatment by monitoring DR progression. In other countries, optometrists are trained to

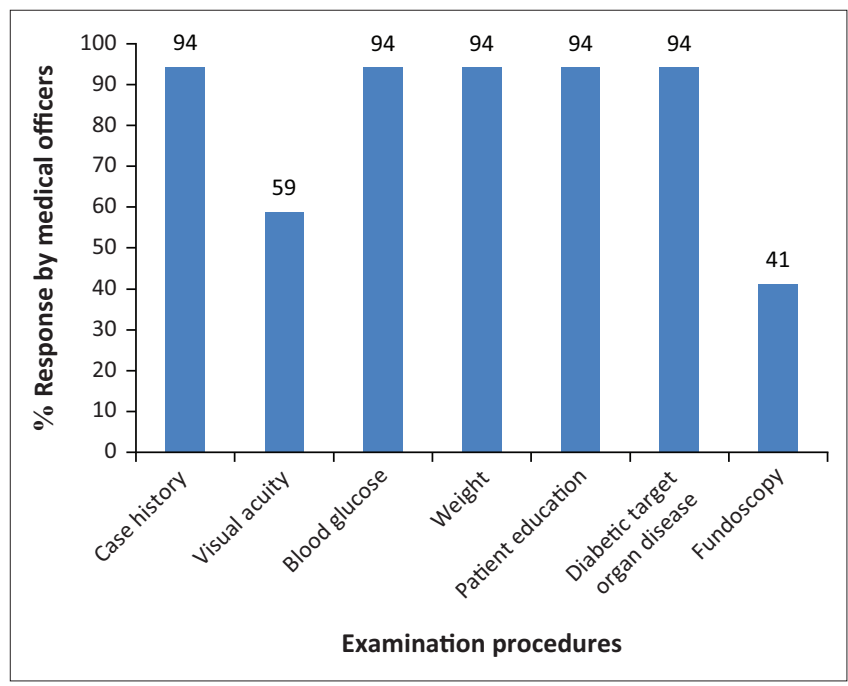

FIGURE 3: Percentage of self-reported competency level of medicals officers (general practitioners) in conducting examination procedures.

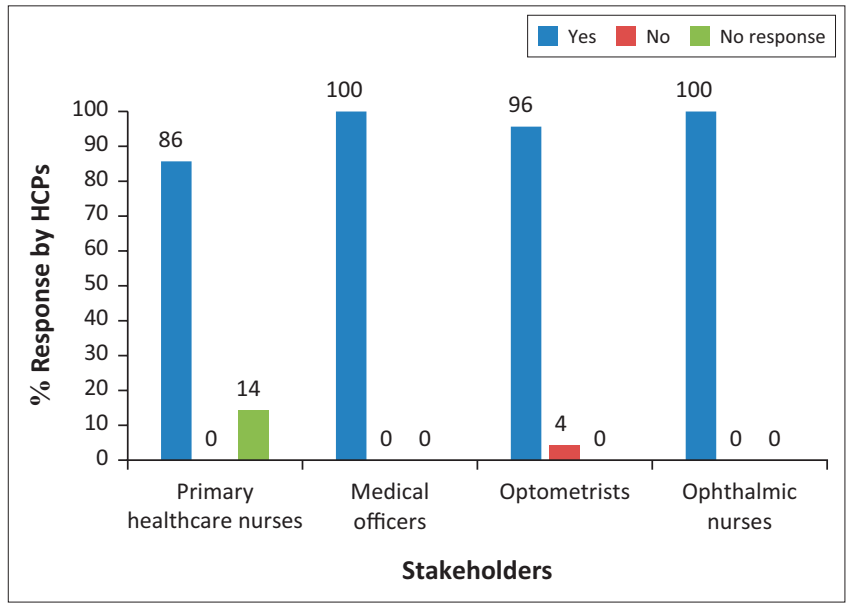

HCP, healthcare practitioners.

FIGURE 4: Percentage of responses by healthcare practitioners in understanding the referral protocol. 
grade DR using different classification systems. ${ }^{15}$ The differences mainly relate to levels of DR grades and to terminology. However, the preferred method of grading DR by the Ophthalmology Society of South Africa is the use of the Scottish Grading Classification System. ${ }^{16}$ Irrespective of which grading classification is preferred, it is important to distinguish between pre-proliferative DR, PDR and diabetic macular oedema (DME) so that surgical treatment can be instituted at an appropriate time in the disease's progression. There is a need to evaluate training at optometry institutions and incorporate a greater emphasis on DR. Because more than half $(52 \%)$ of the optometrists qualified in 2011, graduating with diagnostic skills, with more effective, structured continuing education programmes, their skills in DR screening and grading can be improved. Optometrists could be utilised in conducting training programmes for the ophthalmic nurses and PHC nurses and also in the development of appropriate health promotion material. However, for optometrists to make a significant contribution, more optometry posts need to be created. Screening is usually conducted by optometrists working in the district hospital, and owing to their low numbers in some hospitals, additional screening programmes pose an inconvenience and increase the workload of optometrists. The introduction of a DR training programme will, however, result in more appropriate

TABLE 3: Association between year of qualification of optometrists and grading diabetic retinopathy.

\begin{tabular}{|c|c|c|c|c|c|c|}
\hline \multirow[t]{3}{*}{ Year of qualification } & \multicolumn{4}{|c|}{ Grading DR } & \multicolumn{2}{|c|}{ Total } \\
\hline & \multicolumn{2}{|c|}{ Yes } & \multicolumn{2}{|c|}{ No } & \multirow[t]{2}{*}{$n$} & \multirow[t]{2}{*}{$\%$} \\
\hline & $n$ & $\%$ & $n$ & $\%$ & & \\
\hline Before 2011 & 7 & 63.64 & 4 & 36.36 & 11 & 100 \\
\hline 2011-2016 & 5 & 41.67 & 7 & 58.33 & 12 & 100 \\
\hline Total & 12 & 52.17 & 11 & 47.83 & 23 & 100 \\
\hline
\end{tabular}

Fischer's exact $=0.414$.

DR, diabetic retinopathy.

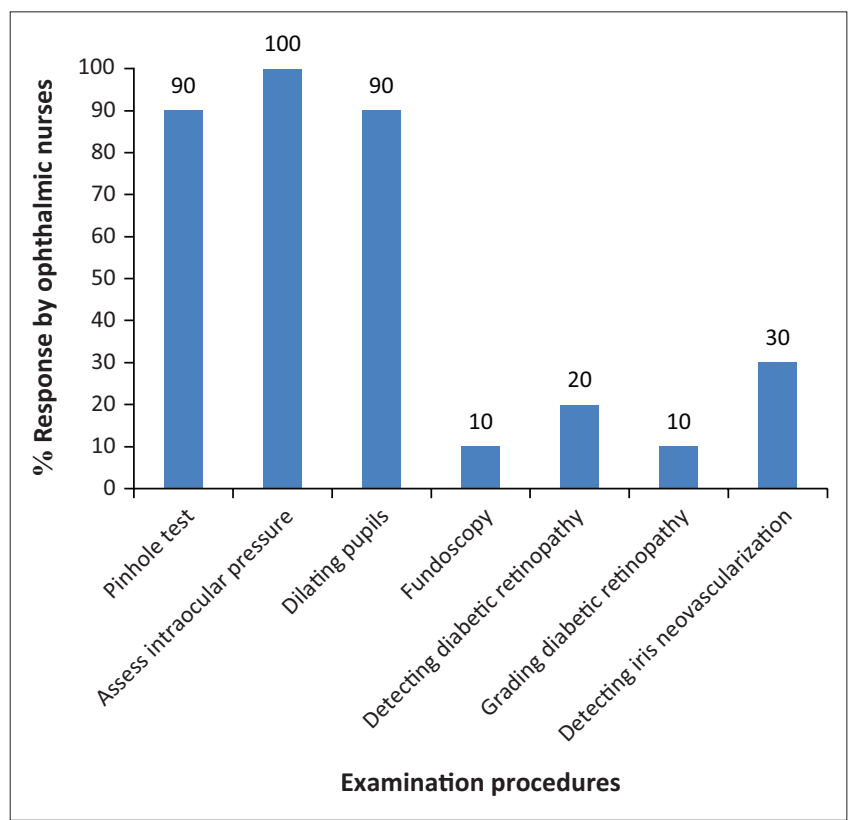

FIGURE 5: Percentage of self-reported competency level of ophthalmic nurses in conducting examination procedures. referrals to ophthalmologists and patients not needing surgical intervention yet will be kept out of busy clinics and monitored by trained graders.

Six optometrists requested modification of the DR screening model (Figure 7). Four recommended involving dieticians, occupational therapists and home-based carers; one optometrist suggested that the ophthalmic nurses grade and monitor DR progression, whereas the other optometrist suggested that ophthalmic nurses and PHC nurses are not required in the structure. In the development process of the DR screening model, endocrinologists also suggested that there was no need for ophthalmic nurses to be involved in a DR screening programme in a resource-constrained setting as too many tiers may lead to confusion and that cost of these tiers versus effectiveness should be considered. Whilst

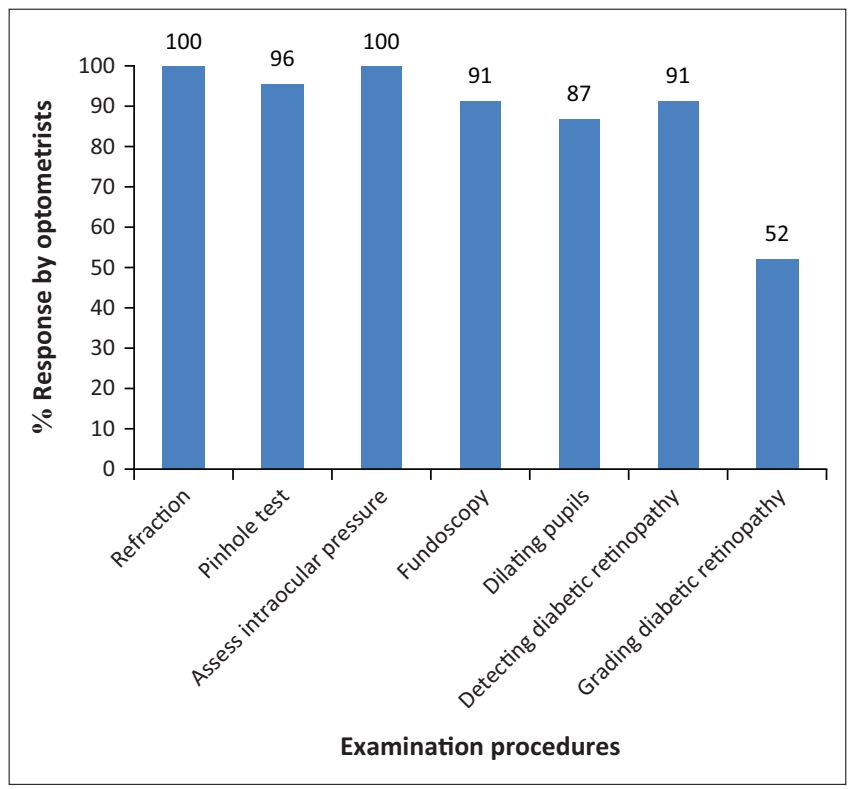

FIGURE 6: Percentage of competency level of optometrists in conducting examination procedures.

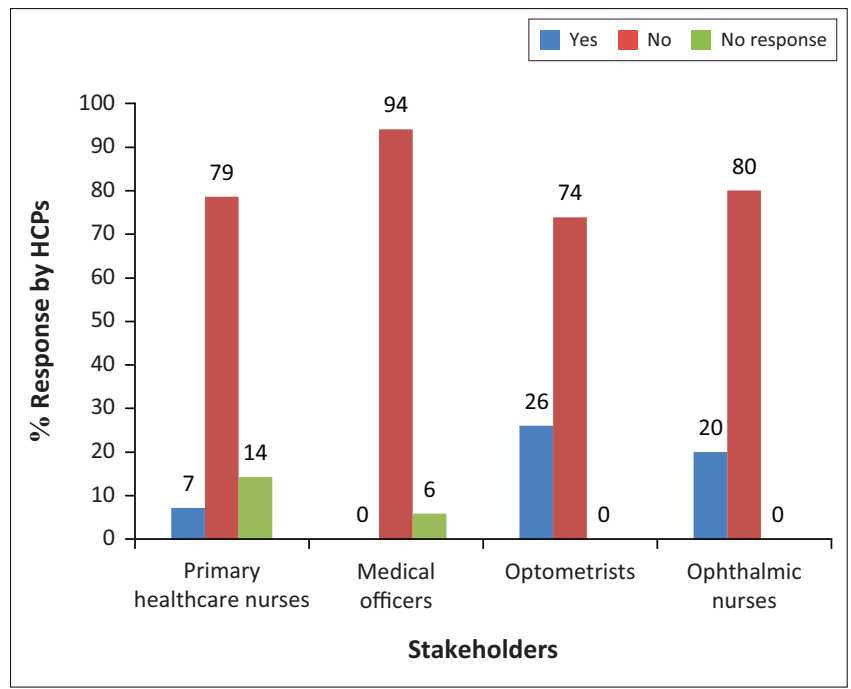

HCPs, healthcare practitioners.

FIGURE 7: Percentage of responses by healthcare practitioners to modify diabetic retinopathy screening protocol. 
the involvement of ophthalmic nurses remains crucial, the reality is that more optometrists than ophthalmic nurses are employed in the public sector in Limpopo province. Ophthalmologists are dependent on optometrists to screen for DR and require the ophthalmic nurses to assist in surgical procedures. Also, the exclusion of PHC nurses will not be beneficial to the DR screening model as PHC nurses are in a position to ensure coordinated care for patients with DM by ensuring regular reviews and medication compliance.

\section{Conclusion}

Based on the assessment of competency level of HCPs, the proposed DR screening model can be implemented without changing the current roles of HCPs in the management of patients with DM in the DHS. However, by introducing training programmes for optometrists to grade DR, involving dietician visits to re-emphasise patient education and by creating more posts for ophthalmic nurses, the DR screening model can be strengthened.

\section{Acknowledgements}

The authors thank the nursing managers in Waterberg clinics for supporting the study enthusiastically; R.F. Segooa, the optometry co-ordinator for Waterberg district, for assistance in facilitating questionnaire distribution to ophthalmic nurses and optometrists; and C.J. van Rensburg from the Medical Research Council for assistance with statistical analysis.

\section{Competing interests}

The authors declare that they have no financial or personal relationships that may have inappropriately influenced them in writing this article.

\section{Authors' contributions}

Z.A. was the project leader and executed meetings with PHC nurses in Waterberg district clinics, collected and captured data, wrote up the research and prepared the draft for publication. K.N. and L.V. reviewed the content of questionnaires for applicability, construct validity and reliability and provided guidance as supervisors to the writeup of the research thesis.

\section{Funding information}

This research received no specific grant from any funding agencies in the public, commercial or not-for-profit sectors.

\section{Data availability statement}

The data collected will be used solely for the purposes of completing my research thesis and in future, articles, and books that will be written by the author.

\section{Disclaimer}

The views and opinions expressed in this article are those of the authors and do not necessarily reflect the official policy or position of any affiliated agency of the authors.

\section{References}

1. Abdool Z, Naidoo K, Visser L. The management of diabetic retinopathy in the public sector of eThekwini district of KwaZulu-Natal. Afr Vision Eye Health. 2016;75(1):a344. https://doi.org/10.4102/aveh.v75i1.344

2. Department of Health. Chronic diseases, disabilities and geriatrics. In: National guidelines - The management of diabetes, type 1 and type 2 in adults at hospital level. Pretoria: Department of Health, 2005; p. 35-36.

3. Shaya FT, Aljawadi M. Diabetic retinopathy. Clin Ophthalmol. 2007;1(3):259-265.

4. Fong DS, Aiello L, Gardner TW, et al. Retinopathy in diabetes. Diabetes Care. 2004;27(Suppl 1):S84-S87. https://doi.org/10.2337/diacare.27.2007.S84

5. Kanski J.J. Clinical ophthalmology. In: Kanski J.J, editor. Retinal vascular disorders. 3rd ed. Oxford: Butterworth-Heinemann, 1994; p. 344-357.

6. Guigui S, Lifshitz T, Levy J. Diabetic retinopathy in Africa: Advantages of screening Postgrad Med. 2011;123(4):119-125. https://doi.org/10.3810/pgm.2011.07.2311

7. Chakrabarti R, Harper CA, Keeffe JE. Diabetic retinopathy management guidelines. Expert Rev Ophthalmol. 2012;7(5):417-439. https://doi.org/10.1586/eop.12.52

8. Murthy GVS, Raman U. Perspectives on primary eye care. Community Eye Health. 2009;22(69):10-11.

9. Kanavus P, Van Den Aardweg S, Schurer W. Diabetes expenditure, burden of disease and management in $5 E \mathrm{EU}$ countries. London: The London School of Economics Health and Political Science; 2012.

10. Baba D, Krishnan M, Bhaskaran S, et al. Prevalence of diabetic retinopathy and correlation with systemic risk factors in type 2 diabetes mellitus in a tertiary care hospital. Sch J App Med Sci. 2015;3(7C):2659-2664.

11. Wykoff CC, Brown DM. Diabetic retinopathy: A team approach to screening, referral and treatment [homepage on the Internet]. Medscape; 2012 [cited 2013 Feb 06]. Available from: http://www.medscape.com/viewarticle/760431_print

12. South African Department of Health. National guideline - Prevention of blindness in South Africa. Pretoria: DOH; 2002.

13. Republic of South Africa. Essential Drugs Programme. Hospital level (Adults) Standard Treatment Guidelines and Essential Medicines List. 3rd ed. Republic of South Africa: National Department of Health; 2012.

14. Antwi R. Ophthalmic nursing: Importance and challenges [homepage on the Internet]. The International Agency for the Prevention of Blindness; 2019. [cited 2019 Aug 5]. Available from: https://www.iapb.org/news/ophthalmic-nursing importance-and-challenges/)

15. Scientific Department, The Royal College of Ophthalmologists. Diabetic retinopathy guidelines: Page 7. London: The Royal College of Ophthalmologists; 2012.

16. Goatman KA, Philip $S$, Fleming $A D$, et al. External quality assurance for image grading in the Scottish Diabetic Retinopathy Screening Programme. Diabetic Med. 2012; 29:776-783. https://doi.org/10.1111/j.1464-5491.2011.03504.x 